Check for updates

Cite this: RSC Adv., 2017, 7, 21780

Received 9th February 2017

Accepted 5th April 2017

DOI: 10.1039/c7ra01681f

rsc.li/rsc-advances

\section{Poly(ethylene terephthalate-co-isophthalate) synthesized via a Sb/Al bimetallic compound catalyst: the effect of the end groups on the properties of polyester}

\begin{abstract}
Fuchen Zhang, (D) Quxia Wang, Lipeng Wang and Yongping Bai*
Herein, poly(ethylene terephthalate-co-isophthalate) (PETI) was synthesized via an ethylene glycol stibium/ $\gamma$-AlOOH composite catalyst and a study was carried out on the structural changes of the end groups of poly(ethylene terephthalate-co-isophthalate) (PETI). Based on this study, the effect of the structural changes of these end groups on the crystallization and optical properties of polyester has been discussed. Moreover, it was found that after the catalytic reaction, the $\gamma-\mathrm{AlOOH}$ and the carboxyl end groups of PETI generated terminal carboxyl aluminum coordination compounds. The composite catalyst generated alkoxy aluminum and alkoxy stibium. The strong nucleating ability of these new end group structures changed the crystallization properties of the polyester. Moreover, the optical properties of the polyester were affected and as a result, a $90.46 \%$ transmittance for PETI was observed.
\end{abstract}

\section{Introduction}

Thermoplastic polyester materials as an important class of polymers bring great convenience to people's life. These materials are widely used in the field of fibers, films, PET bottles, medical materials, and engineering plastics. ${ }^{1-3}$ Stibium catalysts are the most common catalysts in polyester synthesis. Among these, ethylene glycol antimony as a polyester polycondensation catalyst is extensively used in the industry due to its good solubility, high catalytic efficiency, and the stable quality of the polyester produced via it. ${ }^{4}$ In addition, titanium catalysts and tin-based catalysts are being widely used and investigated; however, the titanium catalysts and tin-based catalysts, represented by tetrabutyl titanate and stannous oxalate, respectively, are liable to cause side effects, thereby deteriorating the polyester properties. Thus, these catalysts are continually being improved. ${ }^{5-9}$ It is generally considered that the catalysts for polycondensation reactions form alkoxides or chelates with polyester end groups after the catalytic reaction. However, inorganic catalysts, such as stibium trioxide and germanium dioxide, are believed to remain in the polyester in the in situ state. The residue of these condensation catalysts has a significant impact on the performance of polyester. ${ }^{10,11}$ For example, Aharoni studied why titanium catalysts made PET become dim. He proved that titanium catalysts produce black elemental stibium due to reduction by $\mathrm{CO}$ and $\mathrm{CH}_{4}$, which

School of Chemical Engineering and Technology, Harbin Institute of Technology, Harbin 150001, P. R. China. E-mail: baifengbai@hit.edu.cn; Fax: +86-045186414086; Tel: $+86-0451-86413711$ affects the color of the polyester. ${ }^{12}$ Toufaili studied the reaction mechanism of the stibium-catalyzed polycondensation of poly(ethylene terephthalate), which confirmed that the stibium catalysts react with the hydroxyl end groups of PET to chelate the ligands and also to form stibium alkoxides. ${ }^{13}$ Therefore, the various types of catalysts, even at $0.01-0.05 \mathrm{wt} \%$ in the final polymer, can significantly affect the crystallization properties, byproduct content, and many other properties of thermoplastic polyester. Thus, the study of the catalysts remaining as a residue in polyester is very important. However, existing studies on the catalysts for polyester polycondensation have mainly focused on the catalytic properties, byproduct type and content, PET hue, and other properties. ${ }^{14,15}$ Moreover, there are few studies on the residue fractions or concrete structures of a wide variety of catalysts after the catalytic reaction. However, the influence mechanism of the catalyst residue on the performance of thermoplastic polyester has not been fully investigated.

The most common end groups in thermoplastic polyesters are hydroxyl and carboxyl, which have chemical activity and a complex impact on the polyester properties. The terminal carboxyl group is mainly produced via thermal oxidation and thermal degradation reactions. In these processes, the color of the polyester can be changed. Therefore, the content of the terminal carboxyl group is commonly used to evaluate the degree of the side reactions in the polyester synthesis process. ${ }^{16}$ Furthermore, carboxyl end group can also promote polyester hydrothermal aging, decreasing aging resistance of PET. ${ }^{17,18}$ Terminal hydroxyl and terminal carboxyl groups can be reacted with hydroxyl and carboxyl, respectively, which lead to capping, transesterification or chain-extending reactions. ${ }^{19-22}$ Therefore, 
the content and structure of the polyester end groups have an important impact on the properties of polyester. The metal ions of the catalysts react with the polyester end groups to complete the catalytic reaction; thus, the catalyst may affect the structure of the polyester chain ends after the reaction.

Poly(ethylene terephthalate-co-isophthalate) (PETI), as an important member of the polyester family, is a common thermoplastic polyester that is widely used in films, food packaging, and PET bottles, among other fields. ${ }^{23,24}$ In the previous studies, we investigated in detail the catalytic properties of the composite catalysts and the production of the byproducts in the catalytic synthesis of PETI. The $\mathrm{Sb} / \mathrm{Al}$ composite catalyst consists of ethylene glycol stibium and $\gamma$-AlOOH. We proved that ethylene glycol stibium and $\gamma$-AlOOH have synergetic catalytic properties and the catalytic efficiency is higher when both are used..$^{25}$ In a subsequent study, we unexpectedly found that this composite catalyst had an interesting effect on the crystallization properties and optical properties of PETI. Through further research, these changes were directly linked to the residues generated by the $\mathrm{Sb} / \mathrm{Al}$ composite catalyst after catalytic reaction, which influenced the properties of PETI by changing the structure of the end groups in PETI.

\section{Experimental}

\subsection{Materials}

Pure terephthalic acid (PTA, 99.9\%) was provided by Yizheng GeLin Man Chemical Co. Ltd. Isophthalic acid (IPA, 99.9\%) was purchased from AGIC International Chemical Company (Japan). Ethylene glycol (EG, 99.8\%) was obtained from Jiangsu Yong Feng Chemical Co. Ltd. Ethylene glycol stibium (Sb content of 56-57\%) was supplied by Shanghai Qi Zhi Chemical Co. Ltd. Aluminum diacetate hydroxide and trimethyl phosphate (TEP) were purchased from Aladdin Industrial Corporation (Shanghai, China). The chemicals were of analytical grade unless otherwise indicated.

\subsection{Synthesis of PETI}

A certain proportion of PTA, IPA, and EG were added into a $5 \mathrm{~L}$ polyester synthesis device equipped with a process tower, cooling tower, and a vacuum system. IPA accounted for $0.5 \mathrm{wt} \%$ of the diacid. The esterification reaction was carried out at 230$265{ }^{\circ} \mathrm{C}$. When the actual water yield of the esterification process reached $92 \%$ of the theoretical water yield, the reaction underwent the pre-polycondensation stage. In this stage, the reactor was evacuated to reduce the pressure $(30 \mathrm{~Pa})$ within $1 \mathrm{~h}$ while maintaining the temperature at $270{ }^{\circ} \mathrm{C}$. The final polycondensation reaction was conducted under a vacuum degree less than $20 \mathrm{~Pa}$. The reaction endpoint was examined by a $68 \mathrm{~W}$ motor power. PETI was extruded using nitrogen and was then cut and dried. Before the pre-polycondensation stage, TEP, which accounted for $0.005 \mathrm{wt} \%$ of the diacid, was added as a stabilizer. According to the experimental design, ethylene glycol stibium was added in the esterification stage, whereas $\gamma$ $\mathrm{AlOOH}$ was added either during the esterification stage or before the start of the pre-polycondensation stage (the intrinsic viscosity of PETI obtained by the polycondensation reaction was substantially the same and was in the range from 0.69 to $0.79 \mathrm{dL}$ $\mathrm{g}^{-1}$ (refer to ref. 25)). In the esterification reaction stage, both terephthalic acid and isophthalic acid can react with ethylene glycol to form the esterification products 1 and 2; moreover, the esterification products 1 and 2 can continue to undergo esterification to form the esterification product 3 , which was simultaneously carried out. After the polycondensation reaction, the main body of the PETI copolyester was mainly composed of terephthalic acid, isophthalic acid, and ethylene glycol, and these three types of structural units were randomly arranged (Scheme 1).

\subsection{Characterization}

Crystallization properties. The crystallization temperature was measured using a differential scanning calorimeter (DSC 200 F3, Germany). The procedure along with the operating conditions is as follows: first, the sample was heated from $25{ }^{\circ} \mathrm{C}$ to $280{ }^{\circ} \mathrm{C}$ at the heating rate of $10{ }^{\circ} \mathrm{C} \mathrm{min}^{-1}$, kept at $280{ }^{\circ} \mathrm{C}$ for $3 \mathrm{~min}$, and then cooled down to $30{ }^{\circ} \mathrm{C}$ at the cooling rate of $10{ }^{\circ} \mathrm{C} \mathrm{min}^{-1}$. Finally, the sample was reheated to $280^{\circ} \mathrm{C}$ at the heating rate of $10{ }^{\circ} \mathrm{C} \mathrm{min}^{-1}$. The spherulite growth rate was measured using a polarizing microscope (BX51-P, Japan) and a heating stage (THM600, UK). We observed the growth of spherulites using a CCD camera. The operating conditions and procedure are as follows: the sample was heated to $280{ }^{\circ} \mathrm{C}$ at the heating rate of $10{ }^{\circ} \mathrm{C} \mathrm{min}^{-1}$, kept at this temperature for $3 \mathrm{~min}$ to eliminate the thermal history, and then quickly cooled down to the set crystallization temperature. All the samples were vacuum dried at $140{ }^{\circ} \mathrm{C}$ for 4 hours before carrying out the test.

Optical properties. The polyester was hot pressed using a flat plate and stainless steel mold and quenched by cold water to obtain round sheets with a diameter of $4 \mathrm{~cm}$ and a thickness of 1 $\mathrm{mm}$. Then, the surface of the round sheets was pressed into a mirror surface by a sapphire flat sheet mold for investigating the optical properties. The transmittance and haze were tested by a Transmittance/Haze tester (WGT-2S, China). The transmittance curve and reflectivity were tested by a UV-vis-NIR spectrometer (UV-3600, Japan). In this process, the wavelength interval was $5 \mathrm{~nm}$ and the wavelength range was from 200 to $1000 \mathrm{~nm}$. The polyester had absolute reflectivity, which was measured using an integral ball attachment. The test angle was 8 degrees.

Infrared spectrum test. The PETI sample was dissolved in a chloroform/phenol solution (volume ratio, $3: 2$ ), and then this solution was coated onto $\mathrm{KBr}$ pellets. After the solution was dried, the FT-IR spectra were obtained using a Nicolet iS5 spectrometer (Thermo Scientific, USA) in the wavelength range of $4000-400 \mathrm{~cm}^{-1}$. The number of scans was 32 .

\section{Results and discussion}

\subsection{The effect of $\mathrm{Sb} / \mathrm{Al}$ composite catalyst on the end groups of PETI}

In previous reports, we had studied the influence of $\mathrm{Sb} / \mathrm{Al}$ composite catalyst on the catalytic efficiency and thermal properties of PETI and found that the combined use of ethylene glycol 


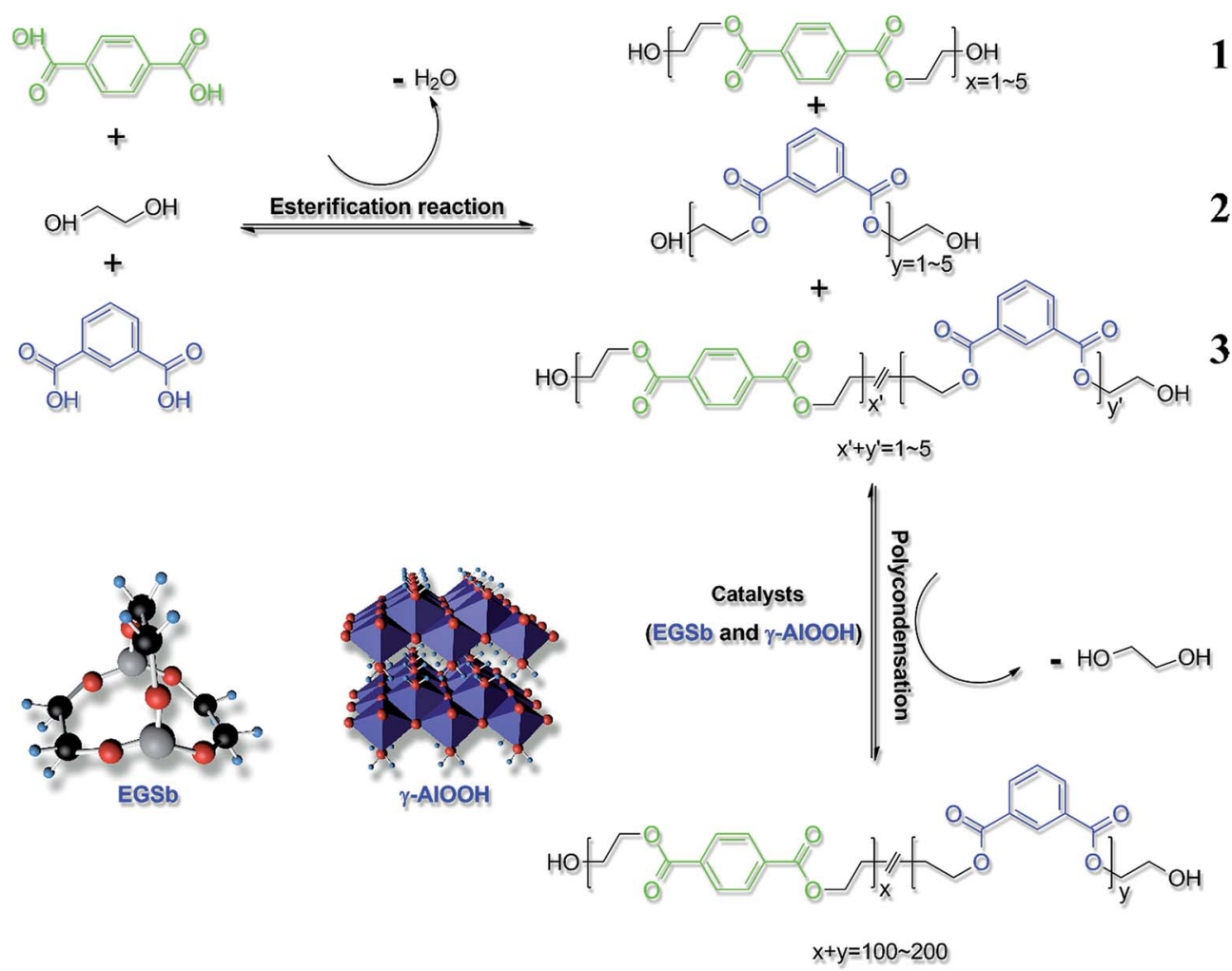

Scheme 1 Synthesis process of poly(ethylene terephthalate-co-isophthalate). (a) 1 and 2 are the esterified products of terephthalic acid and isophthalic acid with ethylene glycol respectively. (b) 3 is an esterified product formed from 1 and 2 .

stibium and $\gamma$-AlOOH can effectively improve the catalytic efficiency of polyester polymerization. ${ }^{25}$ However, in a recent study, we found that there are still many interesting impacts of the catalyst system on PETI; thus, an additional research was conducted.

The amount of the catalyst used in the synthesis of PETI is generally very small, with the amount of PTA in the range of $0.02-$ $0.06 \mathrm{wt} \%$, and the low content of the catalyst and its residues is difficult to detect via conventional methods. We conducted two separate experiments in which we respectively used ethylene glycol stibium and $\gamma$-AlOOH as catalysts, but at $2 \mathrm{wt} \%$ PTA, to synthesize PETI. Therefore, it was easy to characterize the bonds between the catalyst and PETI after the reaction because of the use of a large amount of the catalyst. It was observed that while using the abovementioned amounts of ethylene glycol stibium as a catalyst, the PETI infrared spectrum did not significantly change. In this infrared spectrum, the benzene ring $-\mathrm{C}=\mathrm{C}-$ bending vibration absorption peak at $1613.21 \mathrm{~cm}^{-1}$ and the benzene ring $-\mathrm{C}=\mathrm{C}-$ stretching vibration absorption peak at $1577.79 \mathrm{~cm}^{-1}$ could be observed. However, when $\gamma$-AlOOH was used as a catalyst, the PETI infrared spectrum showed a new absorption peak at $1599.74 \mathrm{~cm}^{-1}$, which is the antisymmetric stretching vibration absorption peak of aluminum carboxylate (COO-) (Fig. 1). This shows that after the reaction, $\gamma$-AlOOH reacts with the carboxyl end groups of PETI to produce terminal carboxyl aluminum salt. When ethylene glycol stibium was used as the catalyst, the carboxylate absorption peak was not observed; thus, ethylene glycol stibium does not react with the carboxyl end groups of PETI to produce carboxylate.
Our previous study has shown that $\gamma$-AlOOH with ethylene glycol can generate ethylene glycol aluminum at high temperature (Fig. 2), which is a type of alkoxy aluminum polymer. Its molecular chains are linked via the alkoxy group. ${ }^{25}$ It is generally believed that in the catalytic process, ethylene glycol salts actually act as a catalyst. Ethylene glycol stibium was in the form of alkoxy stibium in PETI. Similarly, we speculated that ethylene glycol aluminum would be in the form of alkoxy aluminum in PETI.

In summary, $\gamma$-AlOOH with the end groups of PETI generates two structures after the catalytic reaction. One is a terminal carboxyl aluminum salt coordination structure, which is generated by $\mathrm{AlO}_{6}$ and terminal carboxyl (Fig. $3 \mathrm{~A}$ ), ${ }^{26}$ and the other is alkoxy aluminum, which is generated by ethylene glycol aluminum and terminal hydroxyl (Fig. 3B). Moreover, a third structure as alkoxy stibium, which is generated by ethylene glycol stibium and terminal hydroxyl (Fig. 3C), was also observed. The PETI stereomodel shows that the abovementioned three structures can form a cross-linked center structure in polyester. $\left[\mathrm{AlO}_{6}\right]_{n}$ can be combined with several carboxyls; thus, the number of PETI molecular chains bound with it is more than those bound with alkoxy aluminum and alkoxy stibium.

\subsection{The effect of $\mathrm{Sb} / \mathrm{Al}$ composite catalyst on the crystallization properties of polyester}

Further investigation suggested that change in the structure of PETI end groups has a significant effect on the crystallization 


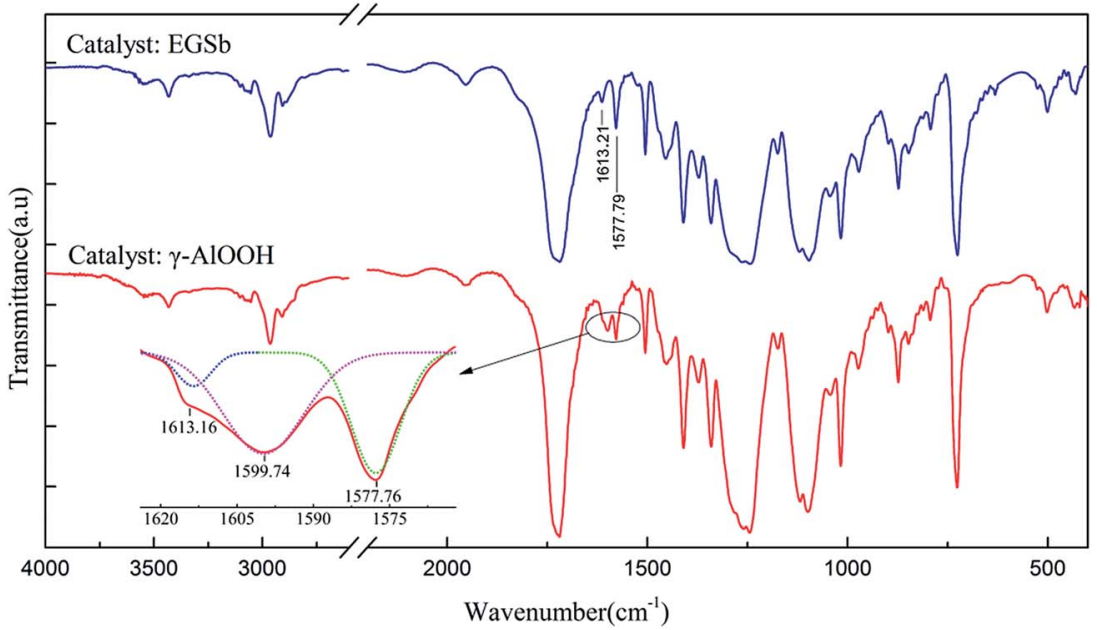

Fig. 1 The infrared spectra of PETI obtained with ethylene glycol stibium and $\gamma-\mathrm{AlOOH}$ as the catalysts.

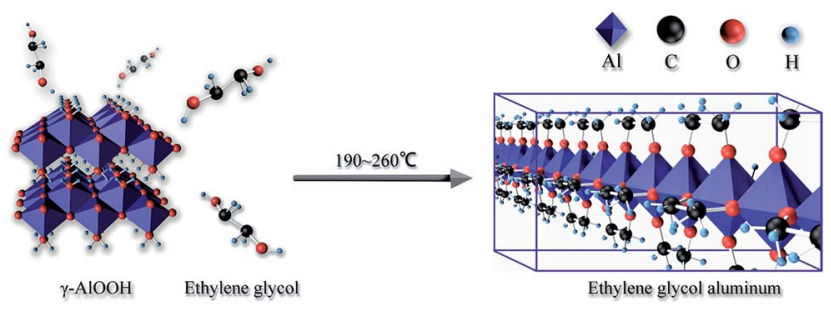

Fig. 2 The structural model of ethylene glycol aluminum generated via $\gamma$-AlOOH and ethylene glycol.

properties, which has a very important impact on the actual use and processability of polyester. When the $\gamma$-AlOOH mass ratio was less than $60 \%$ in the $\mathrm{Sb} / \mathrm{Al}$ composite catalyst, PETI cold crystallization temperature $\left(T_{\mathrm{c}}\right)$ was approximately $135{ }^{\circ} \mathrm{C}$; when the $\gamma$-AlOOH mass ratio was more than $60 \%$, the PETI cold crystallization temperature was approximately $141{ }^{\circ} \mathrm{C}$. These changes were independent of the addition time of $\gamma-\mathrm{AlOOH}$ (Fig. 4a and b). Since less than $7 \%$ of the molecular chains crystallized in PETI, molecular chains rearranged to crystallize in the heating process of DSC, which is the reason for the changes in the cold crystallization temperature. Therefore, the cold crystallization temperature is directly related to the movement ability of the molecular chains. The movement of the molecular chains was more difficult when the $\gamma$-AlOOH mass ratio was more than $60 \%$, which was shown by the changes in the PETI cold crystallization temperature. This may be due to a critical value for the number of alkoxy aluminum and terminal carboxyl aluminum coordination compounds generated by the $\gamma$-AlOOH and PETI
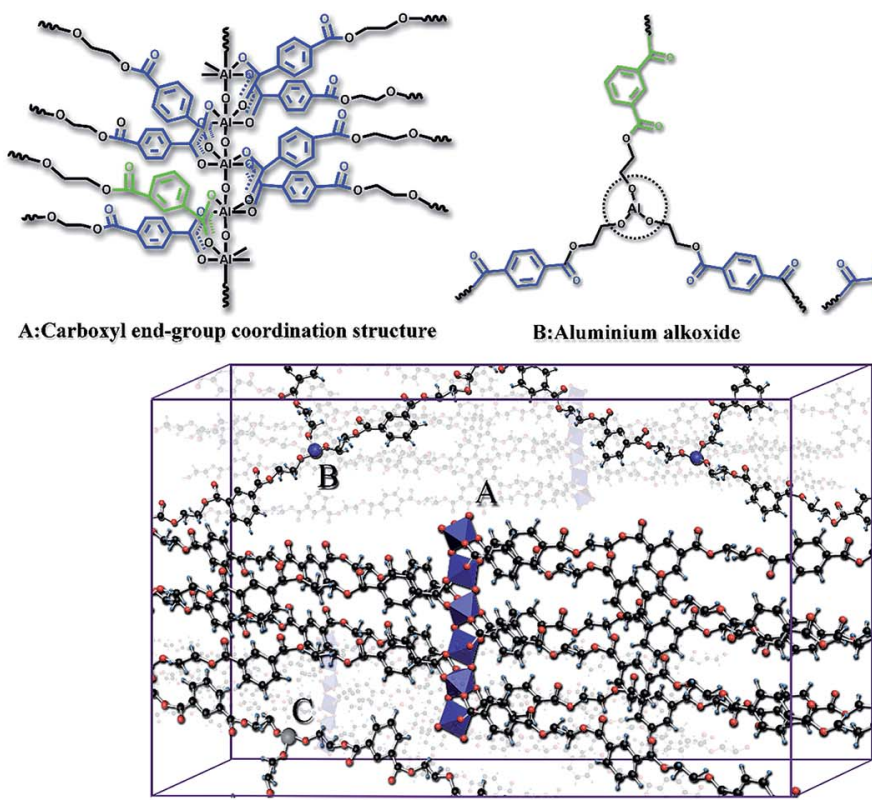

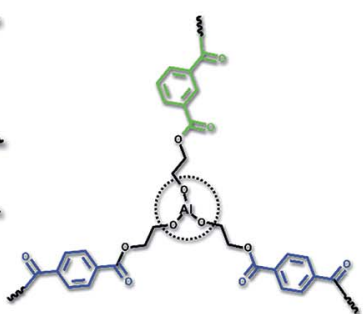

B:Aluminium alkoxide

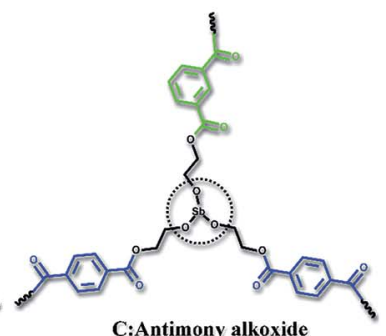

C:Antimony alkoxide

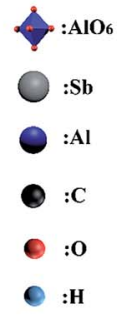

Fig. 3 Three forms of ethylene glycol stibium and the residue of $\gamma$-AlOOH after the catalytic reaction in PETI. 

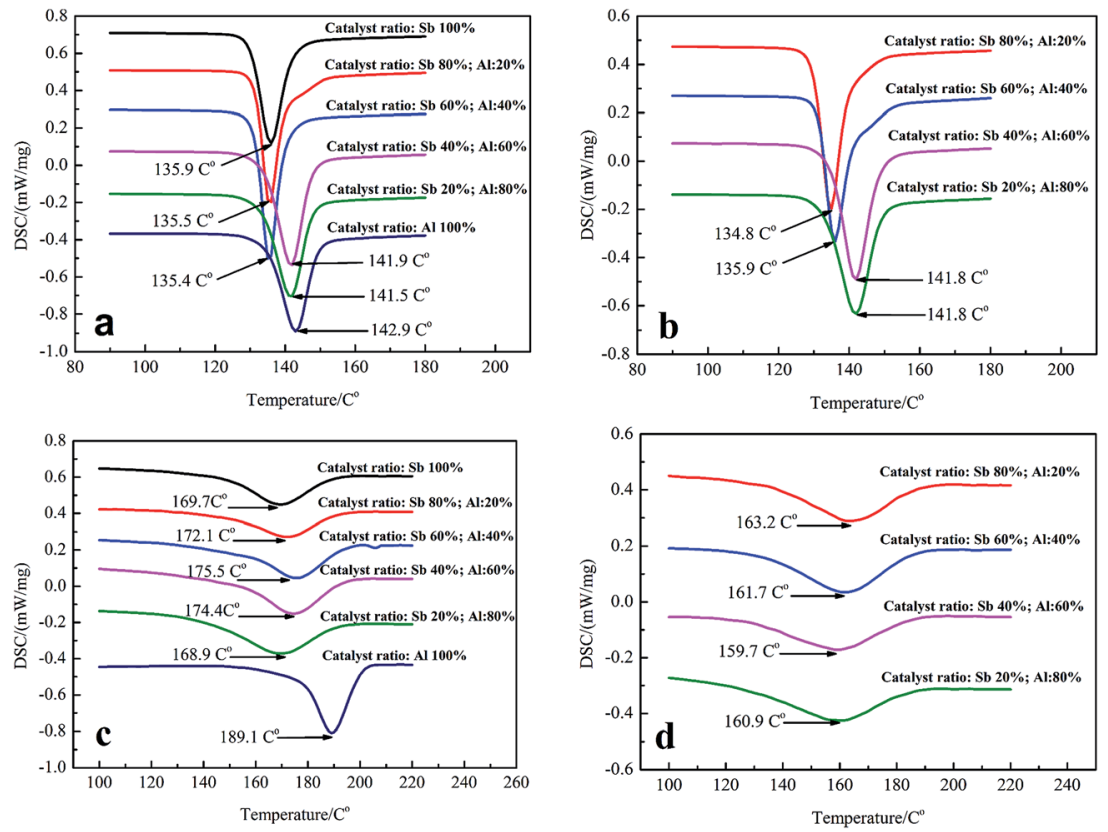

Fig. 4 The effect of $\gamma$-AlOOH addition at the esterification stage and the pre-polycondensation stage on the crystallization temperature of PETI. (i), (a) and (c) are the cold crystallization temperature and thermal crystallization temperature curves of PETI obtained after adding ALOOH at the esterification stage. (ii), (b) and (d) are the cold crystallization temperature and thermal crystallization temperature curves of PETI obtained by adding $\mathrm{ALOOH}$ at the pre-esterification stage.

end groups. It was only when the number of the abovementioned structures exceeded a certain value that the cold crystallization behavior of PETI changed.

Unlike the cold crystallization temperature, the variations in the thermal crystallization temperature $\left(T_{\mathrm{c}}^{*}\right)$ of PETI were more complex (Fig. 4c and d). When $\gamma$-AlOOH was added at the esterification stage, the thermal crystallization temperature first increased and then decreased. The highest thermal crystallization temperature was found when only $\gamma$-AlOOH was used as a catalyst. When $\gamma$-AlOOH was added at the pre-polycondensation stage, the thermal crystallization temperature first decreased and then increased. Thermal crystallization temperature is directly linked to the rate of nucleation and crystal growth; thus, the abovementioned phenomenon has been explained by the nonisothermal crystallization kinetics and crystal growth rate.

To explain the changes of crystallization temperature, the crystallization behavior of PETI was investigated by the Jeziorny method, in which the Avrami equation, which is widely used in the isothermal crystallization analysis, was applied to nonisothermal kinetics, and the resulting parameters were corrected. ${ }^{27}$ On mapping $X_{t}-t-t_{0}$ and $\ln \left[-\ln \left(1-X_{t}\right)\right]-\ln t$ (Fig. 5), the half crystallization time $t_{1 / 2}$, Avrami index $n$, and crystallization rate constant $Z_{\mathrm{c}}$ could be found. Note that the $\mathrm{ln}$ $\left[-\ln \left(1-X_{t}\right)\right]-\ln \left(t-t_{0}\right)$ curve deviated from the expected function at the beginning and end of the PETI crystallization, but maintained a good linear growth in the crystal growth stage.

The calculation results show that the peak crystal growth rate $Z_{\mathrm{c}}$ of cold crystallization was between 1 and 1.21 when $\gamma$-AlOOH was added at the esterification stage, and it was between 0.78 and 0.83 when $\gamma$-AlOOH was added at the pre-polycondensation stage. This proves that PETI, synthesized with $\gamma$-AlOOH added at the esterification stage, had higher cold crystallization rate and higher thermal crystallization growth rate (Fig. $6 a$ and b). End groups of PETI synthesized with $\gamma$-AlOOH added at the esterification stage can form alkoxy aluminum and terminal carboxyl aluminum coordination compounds. These new molecular chains are very similar to star polymers and ionic polymers and thus, the crystallization properties are greatly different from those of general PETI. Because the molecular chains are bound by alkoxy aluminum and $\left[\mathrm{AlO}_{6}\right]_{n}$ coordination structures, the chain segments are so close that it is easier for the chains to be arranged in order. These new structures are also inclined to form nucleating centers, which can promote the increase of the degree of crystallinity of PETI. The number of the abovementioned structures was less when $\gamma$-AlOOH was added at the pre-polycondensation stage and thus the crystal growth rates were much lower.

The half crystallization time $t_{1 / 2}$ shows the rate of crystal growth from the time scale. In terms of the cold crystallization of PETI, with the increasing $\gamma$-AlOOH added, the $t_{1 / 2}$ variation was identical regardless of whether the $\gamma$-AlOOH was added at the esterification or the pre-polycondensation stage. The $t_{1 / 2}$ was lower when the $\gamma$-AlOOH mass ratio was less than $40 \%$ (Fig. 7). Within this range, PETI end groups more easily generated alkoxy aluminum, alkoxy stibium, and terminal carboxyl aluminum coordination compounds, which form the star structures and complex ring structures. These structures may be the nucleating centers and more easily increase the crystallization rate of PETI. However, the variation of the PETI thermal crystallization peak was relatively complex. In terms of common polyester material, the processing is from the melt quenched into an amorphous state; thus, the thermal crystallization properties are very important. The thermal crystallization temperature of PETI synthesized 

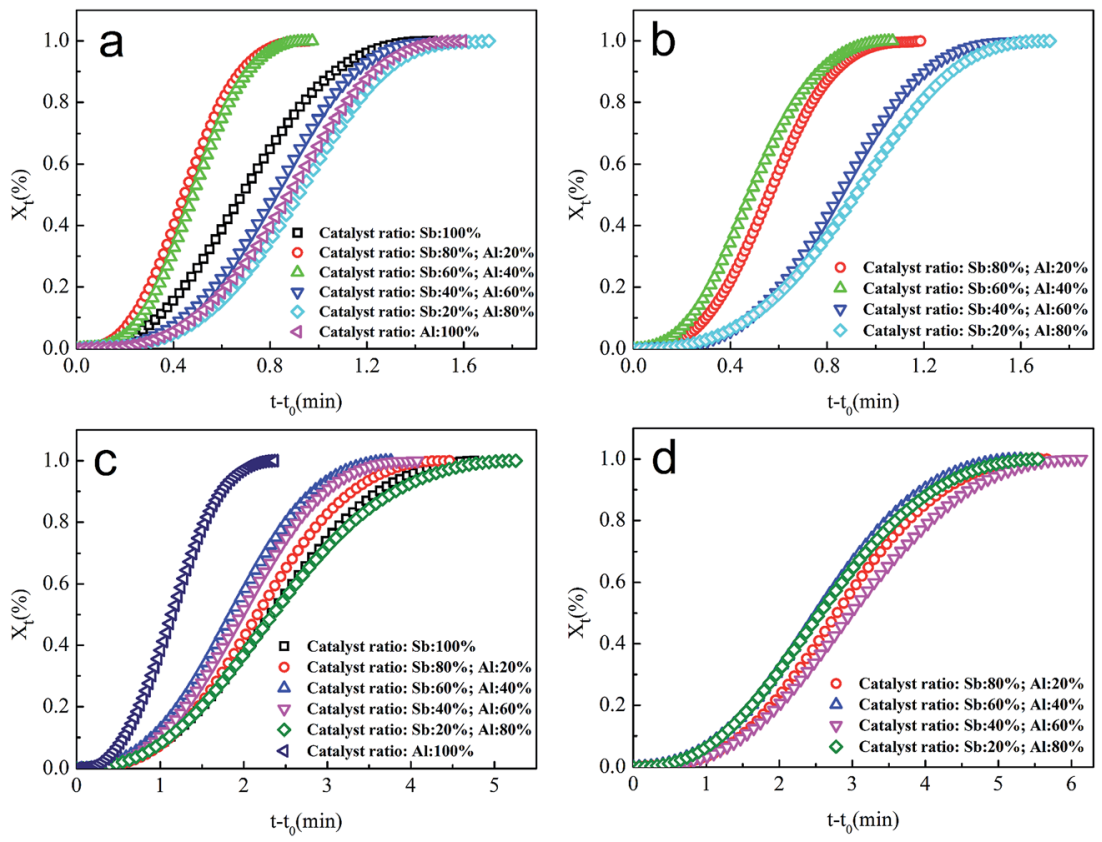

Fig. 5 The $\ln \left[-\ln \left(1-X_{t}\right)\right]-\ln \left(t-t_{0}\right)$ curves of the cold crystallization peak and thermal crystallization peak when $\gamma$-AlOOH was added at the esterification stage and the pre-polycondensation stage. (i), (a) and (b) are the $\ln \left[-\ln \left(1-X_{t}\right)\right]-\ln \left(t-t_{0}\right)$ curves of cold crystallization peak when $\gamma-\mathrm{AlOOH}$ was added at the esterification stage and the pre-polycondensation stage (ii), (c) and (d) are the $\ln \left[-\ln \left(1-X_{t}\right)\right]-\ln \left(t-t_{0}\right) \mathrm{curves}$ of thermal crystallization peak when $\gamma$-AlOOH was added at the esterification stage and the pre-polycondensation stage.

with the composite catalyst in which $\gamma$-AlOOH was added at the pre-polycondensation stage was lower than that when $\gamma$-AlOOH was added at the esterification stage. When adding at the prepolycondensation stage, most of the $\gamma$-AlOOH participated in the catalytic reaction and remained in the PETI. These nanoparticles became crystallization nucleating agents that promoted PET melt crystallization behavior during cooling. However, when added at the esterification stage, $\gamma$-AlOOH in conjunction with ethylene glycol is more likely to generate alkoxy aluminum and terminal carboxyl aluminum coordination compound in a homogeneous material system, which has stronger heterogeneous nucleation ability. When only $\gamma$-AlOOH was used as a catalyst, $t_{1 / 2}$ was the shortest, showing that heterogeneous nucleation was strongest in this case.
The degree of crystallinity of PETI synthesized with the composite catalyst in which $\gamma$-AlOOH was added at the esterification stage was higher than that in which $\gamma$-AlOOH was added at the pre-polycondensation stage (Fig. 8). This further proves that the structure changes in the PETI end groups were influenced by the stibium/aluminum composite catalyst and can significantly affect the crystallization properties. On the one hand, alkoxy aluminum and $\left[\mathrm{AlO}_{6}\right]_{n}$ coordination structures can promote the orderly folding of the chain segments. On the other hand, these become nucleating centers. All these enhance the degree of crystallinity of PETI. The relative content of the new chain structure can be indirectly measured by the variation of the degree of crystallinity.
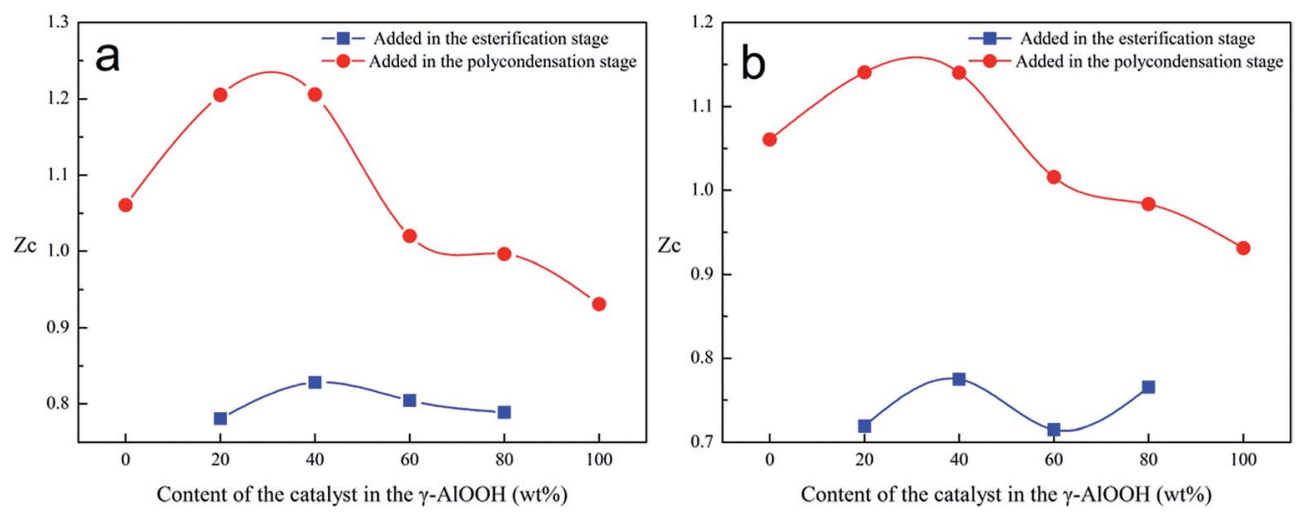

Fig. 6 The variation of crystal growth rate $Z_{c}$ of cold crystallization peak (a) and thermal crystallization peak (b) of PETI synthesized with $\gamma$-AIOOH added in different proportions and different time of addition of catalyst. 

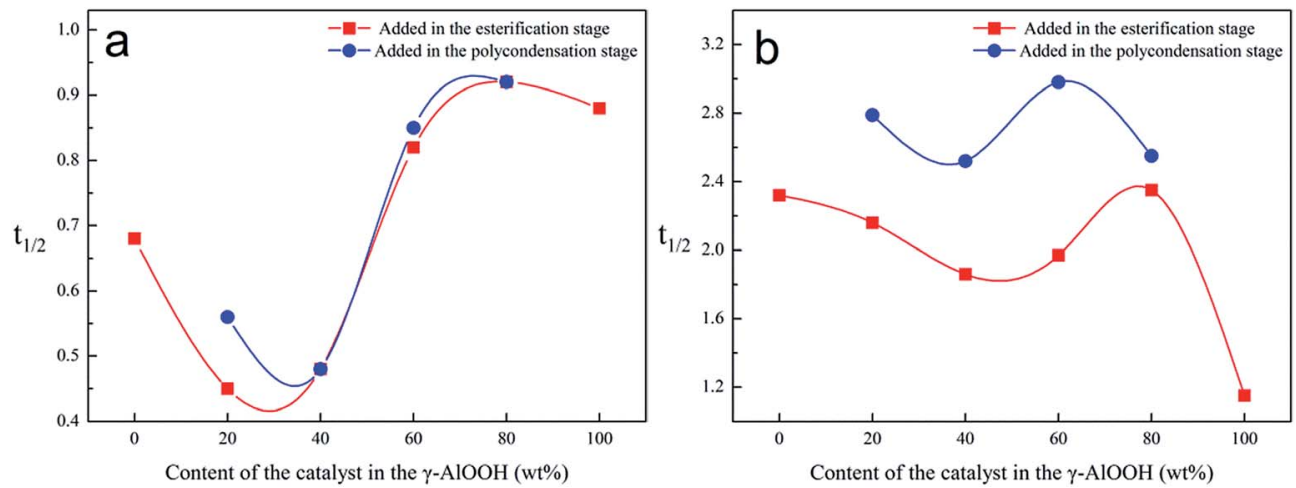

Fig. 7 The effect of different catalyst proportions and the time of addition of catalyst on PETI $t_{1 / 2}$ of cold crystallization (a) and thermal crystallization (b).

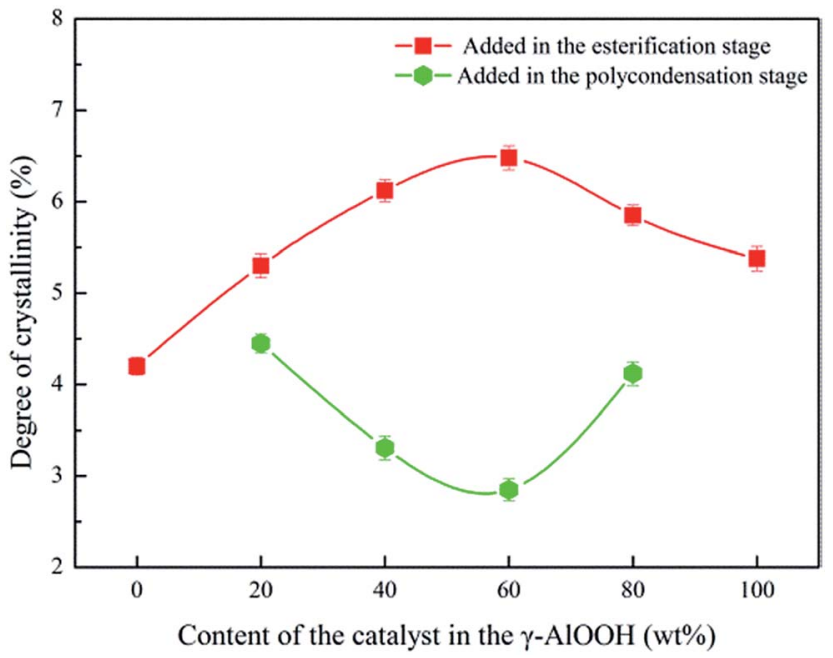

Fig. 8 The effect of different catalyst content on the degree of crystallinity of amorphous polyester.

We observed the PETI spherulite morphology using a polarizing microscope after growing the samples for the same time at $230{ }^{\circ} \mathrm{C}$. The spherulite size of PETI synthesized with only $\gamma$ AlOOH was significantly larger than that of PETI synthesized with only ethylene glycol stibium, and the number of crystal nuclei was lower in PETI synthesized with only $\gamma$-AlOOH. During the crystallization process, $\gamma$-AlOOH and its reaction promoted PETI spherulite growth and same phenomenon was observed with ethylene glycol stibium. The promoting effect of the former is greater than that of the latter, whereas the nucleation rate of the former is lower than that of the latter (Fig. 9a and b). When the mass ratio of the composite catalyst was $\mathrm{Sb}: \mathrm{Al}=80: 20, \gamma$ AlOOH was added at the esterification stage or at the prepolycondensation stage to obtain PETI. The spherulite size of the former was greater than that of the latter, whereas the number of crystal nuclei in the former was lower (Fig. 9c and d), which shows that the heterogeneous nucleation was the main effect in PETI when we added $\gamma$-AlOOH at the prepolycondensation stage. When $\gamma$-AlOOH was added at the esterification stage and its proportion was $20 \%$ in the composite catalyst, PETI had a higher crystal growth rate in the low temperature region (Fig. 10a). This may be due to the new structures formed by alkoxy aluminum and the terminal carboxyl aluminum coordination compound, which can make molecular chains folded to form crystalline regions centered on them. When $\gamma$-AlOOH was added at the pre-polycondensation stage, the crystal growth rates of PETI synthesized with different proportions of the composite catalyst more intensely changed, which may be due to stronger heterogeneous nucleation in these PETI formulations (Fig. 10b).

\subsection{The effect of $\mathrm{Sb} / \mathrm{Al}$ composite catalyst on the optical properties of PETI}

The structural changes on the PETI end groups also have significant impact on the optical properties of PETI, which can improve the transmittance and reduce the haze in a certain range of catalyst formulations. When the mass ratio of $\mathrm{Sb} / \mathrm{Al}$ was $60: 40$, the transmittance of PETI can reach $90.46 \%$ (Fig. 11a). When $\gamma$-AlOOH was added at the esterification stage, the variation of transmittance was roughly the same as that of $t_{1 / 2}$. With the decreasing $t_{1 / 2}$, the transmittance gradually increased, and the transmittance decreased with the increase of $t_{1 / 2}$. This shows that in this situation, $t_{1 / 2}$ is the main factor that affects the change of PETI transmittance. If $t_{1 / 2}$ of PETI is short, crystal growth time is limited; thus, the spherulite size is smaller than that of PETI when $t_{1 / 2}$ is long. Compared with the addition of $\gamma$ $\mathrm{AlOOH}$ at the esterification stage, when $\gamma$-AlOOH was added at the pre-polycondensation stage, $t_{1 / 2}$ of PETI was longer; thus, crystal growth time and the spherulite size increased, leading to a reduction of the transmittance. However, the variation of transmittance was controlled by the crystal growth rate $Z_{\mathrm{c}}$ and the half crystallization time $t_{1 / 2}$, and it was mainly affected by the $Z_{\mathrm{c}}$. These variations also affected the haze of PETI. The haze of PETI was lower when $\gamma$-AlOOH was added at the esterification stage than that when $\gamma$-AlOOH was added at the prepolycondensation stage (Fig. 11b). Note that the transmittance was low and the haze was high when PETI was synthesized only with $\gamma$-AlOOH, indicating $\gamma$-AlOOH still existed as nucleating centers at the nanometer level after the catalytic reaction and led to deterioration of the optical properties. 

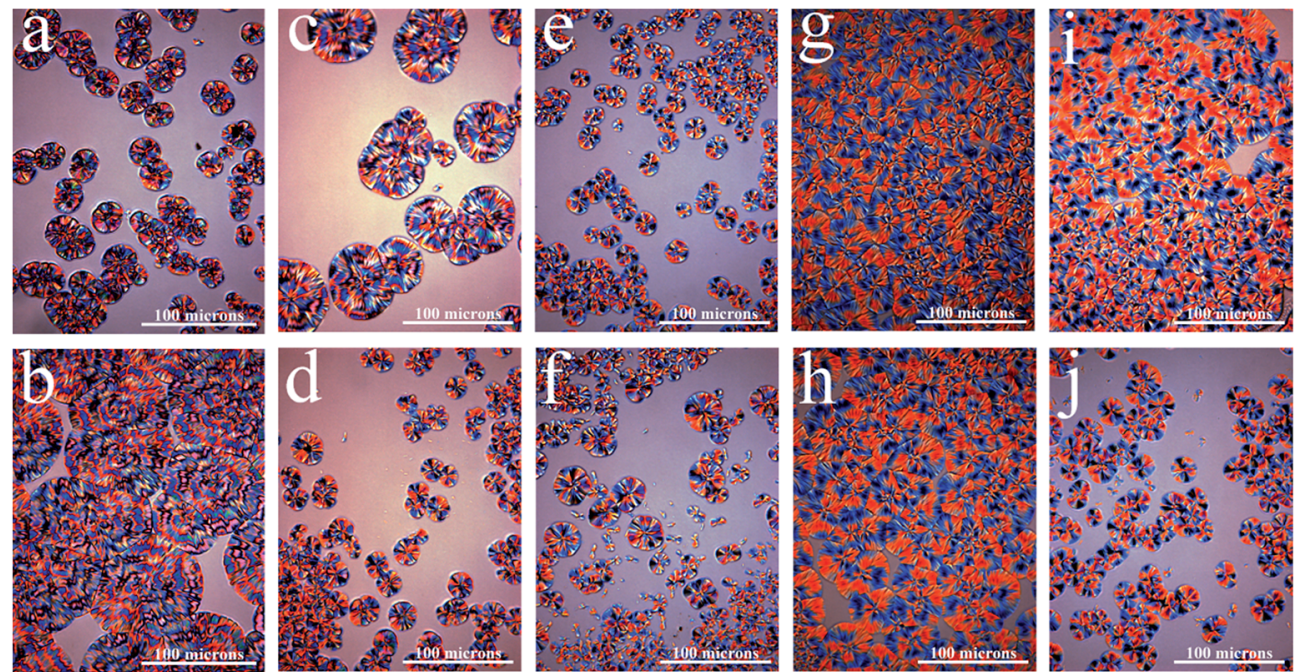

Fig. 9 The spherulitic morphologies of PETI cultured at $230{ }^{\circ} \mathrm{C}$ after $20 \mathrm{~min}$. (a) Using ethylene glycol stibium as the catalyst. (b) Using $\gamma$-AlOOH as the catalyst; addition at the esterification stage. (c), (e), (g), and (i) using the Sb/Al composite catalyst. The catalyst mass ratios were 80 : 20 , $60: 40,40: 60$, and $20: 80$, and $\gamma$-AlOOH was added at the esterification stage. (d), (f), (h), and (j) using the Sb/Al composite catalyst. The catalyst mass ratios were $80: 20,60: 40,40: 60$, and $20: 80$, and $\gamma$-AlOOH was added at the pre-polycondensation stage.
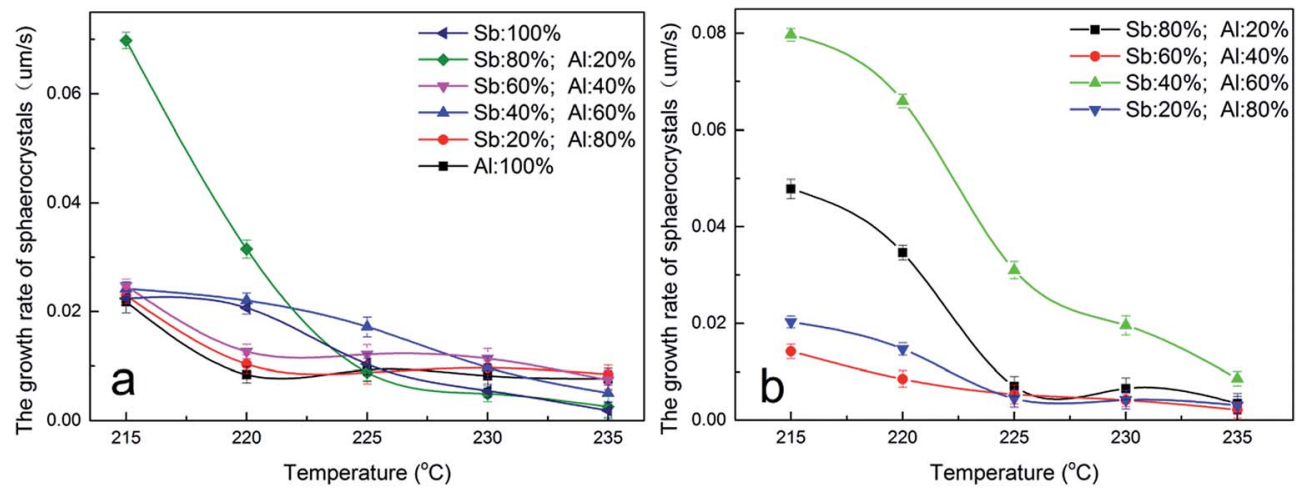

Fig. 10 The spherulite growth rate curve of PETI synthesized with the Sb/Al composite catalyst. $\gamma$-AlOOH was added at the esterification stage (a) and pre-polycondensation stage (b).

Abovementioned transmittance refers to the ratio of the luminous flux through PETI to the total luminous flux, but this cannot accurately explain the specific reasons for the differences in the optical properties of PETI. Through the spectral scanning study, it could be found that there were slight differences in the transmittance curve of PETI in the UV-visible-
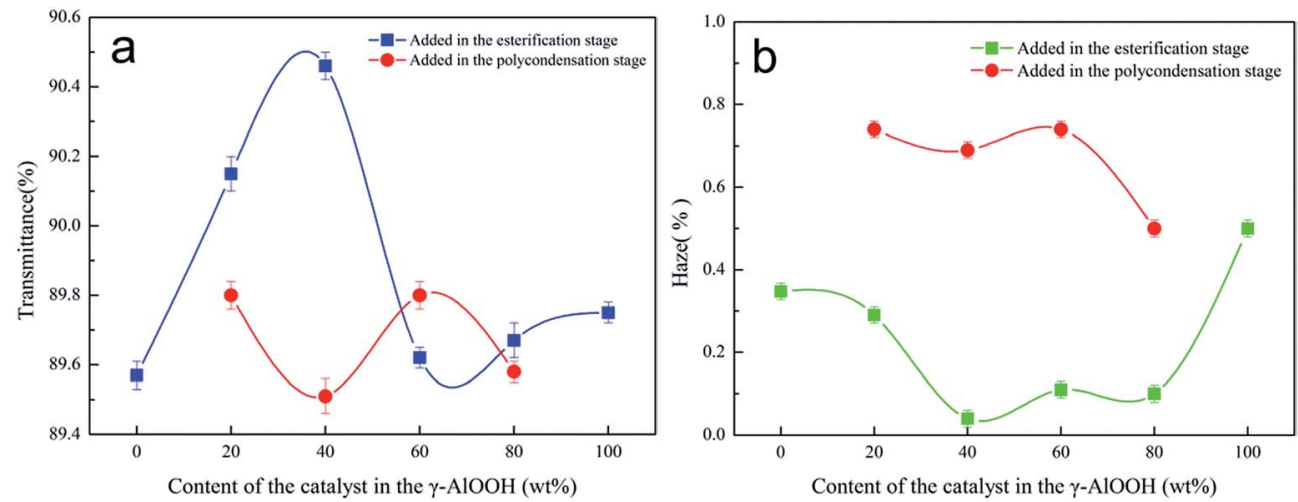

Fig. 11 The effect of the amount of $\gamma-\mathrm{AlOOH}$ added on the transmittance (a) and the haze (b) in the compound catalyst. 

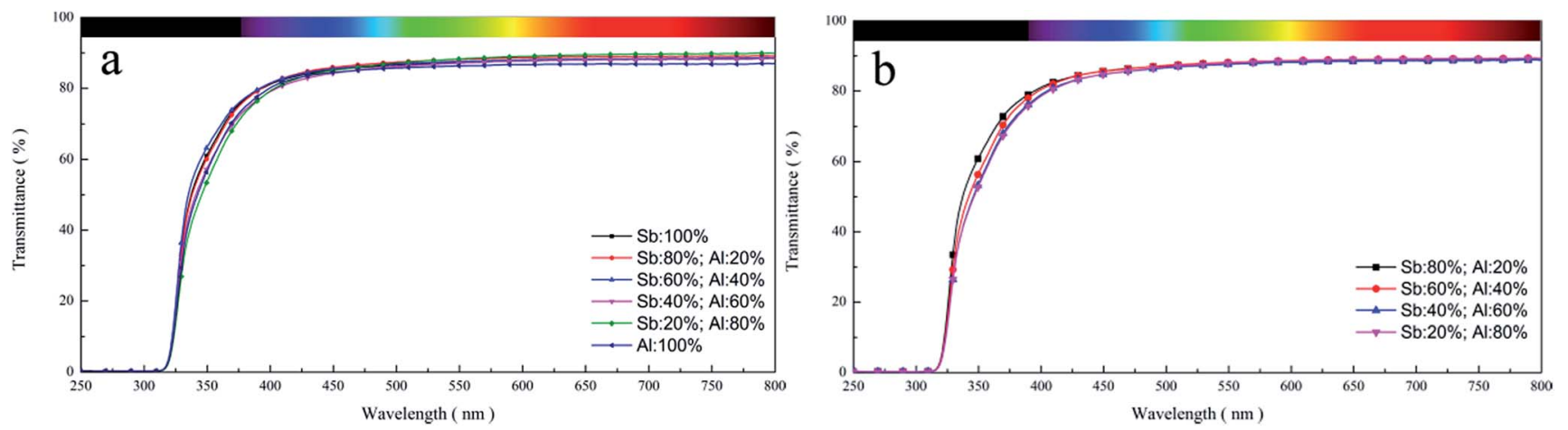

Fig. 12 The UV-vis transmittance spectrum curve of PETI synthesized with the Sb/Al composite catalyst. $\gamma$-AlOOH was added at the esterification stage (a) and pre-polycondensation stage (b).
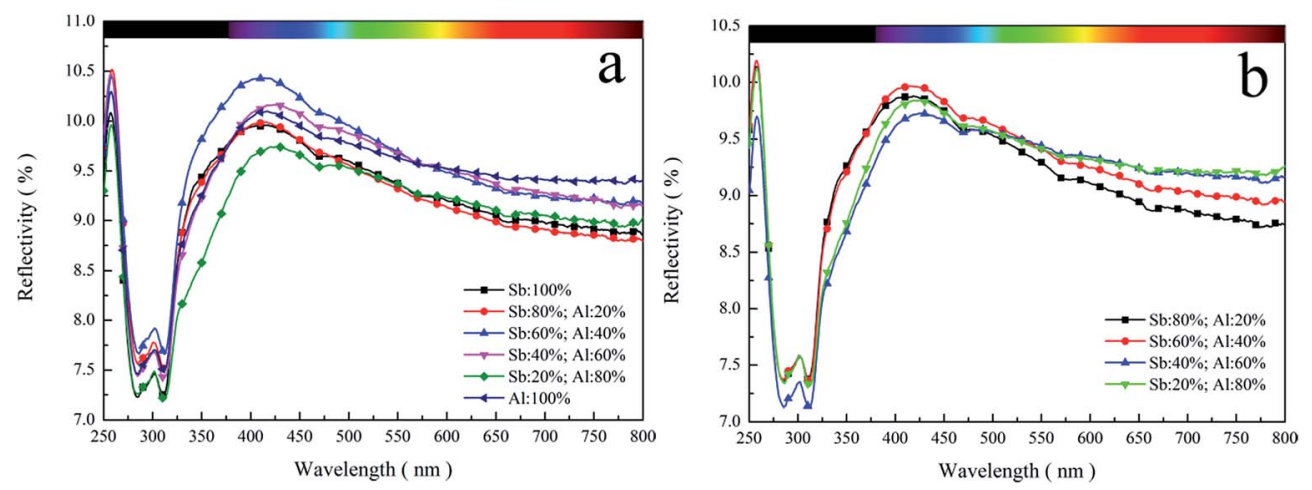

Fig. 13 The UV-vis reflectance spectrum curve of PETI synthesized with the Sb/Al composite catalyst. $\gamma$-AlOOH was added at the esterification stage (a) or pre-polycondensation stage (b).

near infrared region when $\gamma$-AlOOH was added at the esterification stage (Fig. 12a); however, there were slight differences in the $500 \mathrm{~nm}$ wavelength region of the transmittance curve of PETI when $\gamma$-AlOOH was added at the pre-polycondensation stage (Fig. 12b). However, these differences may not result in a very significant effect on the transmittance of PETI.

Furthermore, the light reflection was the main reason for the change in the optical properties of PETI (Fig. 13a and b). PETI absorbs UV light with a wavelength less than $320 \mathrm{~nm}$, and the reflectivity is generally not more than $10 \%$ in the visible region. With the increasing visible light wavelength, reflectivity gradually decreased. The higher the reflectivity of PETI, the lower the transmittance. The fundamental reason for the change of reflectivity of PETI is the change of the degree of crystallinity. The higher the degree of crystallinity, the higher the reflectivity. ${ }^{28}$ The use of the $\mathrm{Sb} / \mathrm{Al}$ composite catalyst changed the degree of crystallinity and thus the reflectivity also changed. Finally, the transmittance of PETI was affected.

\section{Conclusion}

The catalytic reaction to produce poly(ethylene terephthalate-coisophthalate) using $\mathrm{Sb} / \mathrm{Al}$ composite catalyst generates PETI end groups combined with ethylene glycol stibium and $\gamma$-AlOOH. The effect of the combination of the PETI end groups with ethylene glycol stibium and $\gamma$-AlOOH on the crystallization properties and optical properties of PETI was studied. $\gamma$-AlOOH can react with terminal carboxyl of PETI to form terminal carboxyl aluminum coordination compound; moreover, it can also react with terminal hydroxyl of PETI to form alkoxy aluminium and alkoxy antimony. These new terminal derivative structures more easily formed nucleating centers during crystallization and have a stronger ability to crystallize, as compared to heterogeneous nucleating centers, changing the PETI crystallization properties. In addition, these changes in crystallinity altered the optical properties of PETI. Compared with the use of ethylene glycol stibium alone as a catalyst, using the $\mathrm{Sb} / \mathrm{Al}$ composite catalyst increased the transmittance of PETI to $90.46 \%$, the degree of crystallinity of the polyester was changed by the terminated compounds generated by the $\mathrm{Sb} / \mathrm{Al}$ composite catalyst, and the reflectivity was changed, which was the main reason for the change of transmittance. The abovementioned results are expected to be applied in the field of optical polyester synthesis and provide inspiration for the study of new catalysts.

\section{References}

1 V. Tsanaktsis, D. N. Bikiaris, N. Guigo, S. Exarhopoulos, D. G. Papageorgiou, N. Sbirrazzuoli and G. Z. Papageorgiou, RSC Adv., 2015, 5, 74592-74604. 
2 R. Shamsi and G. M. M. Sadeghi, RSC Adv., 2016, 6, 3839938415.

3 L. Diao, K. Su, Z. Li and C. Ding, RSC Adv., 2016, 6, 2763227639.

4 S. M. Biros, B. M. Bridgewater, A. Villeges-Estrada, J. M. Tanski and G. Parkin, Inorg. Chem., 2002, 41, 40514057.

5 C. Di Fiore, B. Leone, C. De Rosa, G. Guerra, V. Petraccone, G. Di Dino, R. Bianchi and R. Vosa, J. Appl. Polym. Sci., 1993, 48, 1997-2001.

6 M. Yin, C. Li, G. Guan, X. Yuan, D. Zhang and Y. Xiao, Polym. Eng. Sci., 2009, 49, 1562-1572.

7 S. Lai, Y. Gao and L. Yue, J. Appl. Polym. Sci., 2015, 132, 41729.

8 X. G. Li, G. Song and M. R. Huang, ACS Sustainable Chem. Eng., 2017, 3, 2181-2195.

9 L. Gustini, C. Lavilla, W. W. Janssen, A. Martínez de Ilarduya, S. Muñoz-Guerra and C. E. Koning, ChemSusChem, 2016, 9, 2250-2260.

10 J. H. Youk, R. P. Kambour and W. J. MacKnight, Macromolecules, 2000, 33, 3594-3599.

11 E. Lawton, Polym. Eng. Sci., 1985, 25, 348-354.

12 S. M. Aharoni, Polym. Eng. Sci., 1998, 38, 1039-1047.

13 F. A. El-Toufaili, G. Feix and K. H. Reichert, J. Polym. Sci., Part A: Polym. Chem., 2006, 44, 1049-1059.

14 J. Yang, Z. Xia, F. Kong and X. Ma, Polym. Degrad. Stab., 2010, 95, 53-58.
15 B. Duh, Polymer, 2002, 43, 3147-3154.

16 F. Pilati, M. Toselli, M. Messori, C. Manzoni, A. Turturro and E. Gattiglia, Polymer, 1997, 38, 4469-4476.

17 D. M. LeMaster and G. Hernández, Macromolecules, 2000, 33, 3569-3576.

18 H. Antheunis, J.-C. van der Meer, M. de Geus, W. Kingma and C. E. Koning, Macromolecules, 2009, 42, 2462-2471.

19 J. Yang, H. Zhu, C. Zhang, Q. Jiang, Y. Zhao, P. Chen and D. Wang, Polymer, 2016, 83, 230-238.

20 F. Samperi, S. Battiato, G. Recca, C. Puglisi and R. Mendichi, Polymer, 2015, 74, 108-123.

21 J. S. Kollodge and R. S. Porter, Macromolecules, 1995, 28, 4089-4096.

22 A. Gallardo, J. San Roman, P. J. Dijkstra and J. Feijen, Macromolecules, 1998, 31, 7187-7194.

23 A. Martínez de Ilarduya, D. P. Kint and S. Muñoz-Guerra, Macromolecules, 2000, 33, 4596-4598.

24 N. C. Karayiannis, V. G. Mavrantzas and D. N. Theodorou, Macromolecules, 2004, 37, 2978-2995.

25 F. Zhang, H. Kang, Y. Bai, B. Jiang, Y. Huang and L. Liu, RSC Adv., 2016, 6, 67677-67684.

26 T. Loiseau, C. Volkringer, M. Haouas, F. Taulelle and G. Férey, C. R. Chim., 2015, 18, 1350-1369.

27 A. Jeziorny, Polymer, 1978, 19, 1142-1144.

28 J. Yoon, R. T. Mathers, G. W. Coates and E. L. Thomas, Macromolecules, 2006, 39, 1913-1919. 